Beim „Bergfest“ berichteten die Mentees

\title{
Die Besten gehören ins Netz
}

„Die Besten gehören ins Netz“ - das ist nicht nur das Motto des Healthcare Frauen e.V., sondern zugleich Anspruch und Ansporn an die Mitglieder des exklusiven Businessnetzwerks. An der diesjährigen Frühjahrstagung der Healthcare Frauen (HCF) Ende April in Köln nahmen 80 TopManagerinnen aus dem Gesundheitswesen teil. Das Netzwerk vereint derzeit 66 Frauen aus der 1. und 2. Führungsebene von Gesundheitsunternehmen und wird dabei aktiv von 4 Fördermitgliedern unterstützt.

„Unser Ziel ist es, die Homogenität in den Führungsetagen des Gesundheitswesens aufzubrechen und mehr Frauen in Führungspositionen $\mathrm{zu}$ bringen. Innerhalb unseres Netzwerks legen wir viel Wert auf Individualität und Authentizität“, erklärt Dr. Vanessa Conin-Ohnsorge, 1. Vorsitzende des Healthcare Frauen e.V., in ihrer Begrüßung. Ein Höhepunkt der Veranstaltung war der Vortrag „Wie können die
Hürden der verschiedenen Unternehmenskulturen überwunden werden, um den Frauenanteil in Führungsetagen zu erhöhen?" von Prof. Dr. Martina Schraudner, Leiterin des Center for Responsible Research and Innovation der FraunhoferGesellschaft. Sie verdeutlichte darin die wichtige Bedeutung der Unternehmenskultur für die Karrierechancen von Frauen. „Um den Anteil von Frauen in Führungspositionen zu erhöhen, ist ein umfassender Kulturwandel in Unternehmen notwendig“, so Schraudner. Sie betonte, dass die für mehr Frauen in Führungspositionen notwendigen Kulturveränderungen Frauen und Männer gleichermaßen adressieren müssen, damit beide $\mathrm{Ge}$ schlechter von den Vorteilen der Kulturveränderung profitieren können. Die Aktivitäten für mehr Frauen in Führungspositionen dürfen sich zudem nicht auf Einzelmaßnahmen beschränken, sondern müssten Teil einer organisationsspezifischen Strategie sein. des aktuellen Mentoring-Jahrgangs von ihren bisherigen Erfahrungen und Erfolgen. Zum Ausklang der Veranstaltung fanden sich alle Teilnehmer zum Get-together zusammen. Diese Gelegenheit nutzten auch viele Gäste mit Interesse an einer Mitgliedschaft bei den Healtcare Frauen oder an einer Teilnahme am HCFMentoringprogramm.

Der Healthcare Frauen e.V. ist die erste und einzige Businessplattform für führende Managerinnen im deutschsprachigen Gesundheitswesen. Erfahrene Frauen aus verschiedenen Disziplinen der Healthcare-Branche treffen sich zum fachlichen, gesellschaftlichen, persönlichen und politischen Austausch, setzen zukunftsweisende Impulse und etablieren effektive Strukturen, führen meinungsbildende Seminare und Vorträge durch, organisieren jährlich laufende Mentoring-Programme, platzieren die nächste Generation von weiblichen Vordenkerinnen und starken Entscheiderinnen im Markt.

Nach einer Pressemitteilung der Healthcare Frauen e.V., Bodenheim Internet: www.healthcare-frauen.de 